\title{
Probing the in-air growth of large area of 3D functional structures into a 2D supramolecular nanoporous network $\dagger$
}

\author{
Romain Brisse, (D) abc Dominique Guianvarc'h, (D) ${ }^{b}$ Christelle Mansuy, (D) ${ }^{b}$ \\ Sandrine Sagan, (DD b ${ }^{\mathrm{b}}$ David Kreher, ${ }^{a}$ Lydia Sosa-Vargas, (D) a Lydia Hamitouche, ${ }^{a}$ \\ Vincent Humblot, (D) ${ }^{d}$ Imad Arfaoui, (D) ${ }^{c}$ Vanessa Labet, (D) ${ }^{c}$ Céline Paris, ${ }^{c}$ \\ Christophe Petit (D) *c and André-Jean Attias (D) *ae
}

\begin{abstract}
Surface-confined host-guest chemistry at the air/solid interface is used for trapping a functionalized 3D Zn-phthalocyanine complex into a 2D porous supramolecular template allowing the large area functionalization of an $\mathrm{sp}^{2}$-hybridized carbon-based substrate as evidenced by STM, resonance Raman spectroscopy, and water contact angle measurements.
\end{abstract}

Supramolecular self-assembly of building blocks (tectons) at surfaces has emerged as a powerful tool to pattern well-organized monolayers on the nanoscale. ${ }^{1}$ The formation of two-dimensional (2D) supramolecular nanostructures is achieved either under ultrahigh vacuum (UHV) or at the liquid/solid interface. ${ }^{2}$ To go beyond the well-mastered decorative $2 \mathrm{D}$ supramolecular self-assembly, the next challenge is the realization by an easy process of large functional surfaces in view of practical applications. ${ }^{3}$

Regarding the issue of dealing with functionalization, two main strategies have been developed: (i) forming 2D surfaceconfined supramolecular nanoporous networks and taking advantage of their potential for hosting guests and confined phenomena. ${ }^{4}$ Several functionalities have been evidenced, ranging from the selective adsorption of guest molecules, ${ }^{5}$ to the guestinduced hierarchical (multicomponent) nanostructures, ${ }^{6}$ control of the dynamic properties of trapped species, ${ }^{7}$ site-selective immobilization of guest molecules in periodically functionalized host cavities, ${ }^{8}$ photo-responsive host-guest systems, ${ }^{9}$ and cavityconfined supramolecular coordination. ${ }^{10}$ However, since the above

\footnotetext{
${ }^{a}$ IPCM, UMR CNRS-Sorbonne Université 8232, 4 Place Jussieu, 75005 Paris, France. E-mail: andre-jean.attias@sorbonne-universite.fr

${ }^{b}$ Sorbonne Université, École Normale Supérieure, PSL University, CNRS, Laboratoire des biomolécules, LBM, 75005 Paris, France

${ }^{c}$ MONARIS, UMR CNRS-Sorbonne Université 8233, 4 Place Jussieu, 75005 Paris, France. E-mail: christophe.petit@sorbonne-université.fr

${ }^{d}$ LRS, UMR CNRS-Sorbonne Université 7197, 4 Place Jussieu, 75005 Paris, France

${ }^{e}$ UMI Building Blocks for Future Electronics, CNRS - Sorbonne Université - Yonsei University - Ewha Womans University, 50 Yonsei-ro Seodaemun-gu, Seoul, Korea. E-mail:aj-attias@yonsei.ac.kr

$\dagger$ Electronic supplementary information (ESI) available: Synthetic details, experimental details, Raman spectroscopy, DFT modeling, and WCA images. See DOI: $10.1039 / \mathrm{c} 8 \mathrm{cc} 06125 \mathrm{~d}$
}

works focused only on 2D components, the interactions with the environment are limited to the upper surface. (ii) This is why to go further in the functionalization, it is mandatory to develop interactions in the dimension perpendicular to the surface. ${ }^{11}$ To address this issue, several approaches have been proposed, based on covalent or noncovalent 3D structures able to project into the third dimension, displaying a functionality. Regarding the covalent 3D tectons, we can mention the triazatriangulenium ion (TATA)-based platform ${ }^{12}$ and Janus tecton platform ${ }^{13}$ developed for possible applications in the fields of molecular machines and photonics, respectively. With respect to noncovalent tectons, porphyrins (P) and phthalocyanines $(\mathrm{Pc})$ are interesting building blocks for obtaining surface-confined self-assembly of 3D functional tectons by metal complexation or axial ligation. ${ }^{14}$ Indeed, it has been demonstrated that the macrocycle unit predominantly adsorbs parallel to the substrate plane, whereas the coordination allows for the projection in the direction perpendicular to the substrate plane. This has been reported for multi-decker lanthanide porphyrin/phthalocyanine sandwich complexes explored as molecular magnets and rotors on the one hand, ${ }^{15}$ and zinc porphyrin ( $\left.\mathrm{ZnP}\right)$ derivatives coordinated with a pyridine-based axial ligand (4-methoxypyridine, or 3-nitropyridine) on the other hand. ${ }^{16}$ Among all the above approaches, mounting on a metalated tetrapyrrole macrocycle an appropriately functionalized axial ligand appears very promising due to the versatile character and ease of the coordination reaction. However, the macrocycles were derivatized to steer the supramolecular selfassembly on the substrate on the one hand, and the coordination occurred on an already metallo-P covered surface on the other hand.

In previous works, ${ }^{17}$ we demonstrated that $1,3,5$-tristyrylbenzene derivatives substituted in positions 3 and 5 by alkoxy peripheral chains presenting $n$ carbon atoms (TSB3,5-C $\mathbf{C}_{n}$ ) (Scheme S1, ESI $\dagger$ ) are able to form, at the liquid/solid interface, by van der Waals interactions between the alkyl chains on highly oriented pyrolytic graphite (HOPG), surface-confined nanoporous honeycomb networks able to act as 2D molecular sieves. More particularly, it was shown that underivatized phthalocyanine $(\mathrm{Pc})$ could be immobilized in the $\mathbf{T S B}, \mathbf{5}-\mathbf{C}_{\mathbf{1 2}}$ 


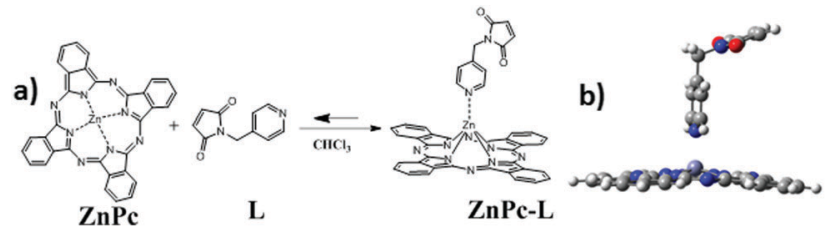

Scheme 1 (a) Complexation of $\mathrm{ZnPc}$ with $\mathrm{L}$ in chloroform and (b) modeling of the chemical structure of the complex by DFT calculations (see the ESI $)$ ).

cavities. ${ }^{18}$ Therefore, we propose to combine the cavity surfaceconfined host-guest recognition and the metallo-Pc axial ligation approaches to immobilize standing-up 3D functional guest structures into the voids of a 2D host template. ${ }^{19}$ Moreover, we provide a way for large area patterning and the associated characterization on different scales in air.

Here, we report the growth, by a drop-casting method at the air/HOPG interface, of large area (up to $\mathrm{mm}^{2}$ ) 3D structures from a 2D porous template and the multiscale characterization by scanning tunneling microscopy (STM), Raman spectroscopy, and contact angle measurements. With this aim, we first synthesized a 3D functional tecton (ZnPc-L) consisting of a pyridine-based ligand (L) functionalized with a maleimide function and mounted on a Zn-phthalocyanine (ZnPc) (Scheme 1). The maleimide function was chosen to allow further thiol-Michael addition click reaction widely used for biomolecular synthesis and labelling of biomolecules. ${ }^{20}$ Our large area patterning process consists of the following sequences: growth of an ordered $2 \mathrm{D}$ porous network by drop-casting from a TSB3,5- $\mathbf{C}_{\mathbf{1 2}}$ solution in chloroform $\left(\mathrm{CHCl}_{3}\right)$, solvent evaporation, and subsequent drop-casting, on the dry host network film, of a 3D ZnPc-L solution in $\mathrm{CHCl}_{3}$, to finally trap them in the template pores. For comparison, similar experiments were performed with planar ZnPc. After evaporation of the solvent, STM images at the air/HOPG interface only provide information of the functionalized surface organization. Therefore, to get some chemical information about the TSB3,5- $\mathbf{C}_{\mathbf{1 2}}$ pores filling with ZnPc or ZnPc-L molecules, the present work explores the use of Raman spectroscopy and contact angle measurements. By combining all the above techniques as well as DFT calculations, we demonstrate that the 3D tectons build up perpendicular to the substrate projecting the maleimide function in a parallel plane above the surface. Unexpectedly, the adlayer hydrophilicity can be tuned depending on the guest molecules.

As an axial ligand, we initially targeted $N$-(4-pyridyl)maleimide by reacting maleimide with 4 -aminopyridine (Scheme S2a, ESI $\dagger$ ). ${ }^{21}$ However, this attempt failed due to the propensity of maleic anhydride to polymerize in the presence of pyridine. ${ }^{22}$ Protecting the maleimide double bond, via the well-known thermoreversible Diels-Alder (DA) reaction, ${ }^{23}$ gave also unreproducible results and did not permit prevention of the polymerization reaction (Scheme S2b, ESI $\dagger$ ), which could be promoted by the high basicity of the pyridine ring in 4 -aminopyridine $\left(\mathrm{p} K_{\mathrm{a}}=9.2\right)$. This is why the ligand $\mathbf{L}$ was targeted (Scheme S3, ESI $\dagger$ ), the synthetic route combines the use of the less basic 4-aminomethylpyridine $\left(\mathrm{p} K_{\mathrm{a}}=4.3\right)$ with the protection/deprotection of the maleimide double bond via a DA/retro-DA reaction (see Experimental details in the ESI $\dagger$ ).
The coordination of ZnPc with ligand $\mathbf{L}$ was operated in solution and monitored by UV/visible spectroscopy (Fig. S1, ESI $\dagger$ ). Chloroform was chosen as the solvent, owing to its poorly coordinating capabilities and so as to maximize the coordination rate of $\mathbf{L}$ with $\mathbf{Z n P c}{ }^{24}$ The obtained solution contained monomeric ZnPc as evidenced by the shape of the UV-visible spectrum (sharp Q-band at $671 \mathrm{~nm}$ and absence of $\mathrm{H}$ or $\mathrm{J}$ shoulders). ${ }^{25}$ The progressive addition of aliquots of $\mathbf{L}$ to the $\mathbf{Z n P c}$ solution induced batho- and hypochromic effects of the Zn-Pc Q-band. In agreement with the literature, ${ }^{26}$ this was attributed to the formation of the ZnPc-L complex. Saturation was reached at about 150 eq., confirming the formation of the ZnPc-L complex with the maximum rate.

In view of potential applications, it is necessary to prove the functionalization on a large scale. To address this issue, the use of complementary techniques is needed. First, the supramolecular self-assembly was investigated by STM. To circumvent the complexification induced by working under UHV or at the liquid/solid interface, large-scale $\left(200 \times 200 \mathrm{~nm}^{2}\right.$ up to $\left.400 \times 400 \mathrm{~nm}^{2}\right)$ STM images were recorded at the air/HOPG interface. With this aim, some representative STM images recorded at different spots of the host nanoporous molecular monolayer and the same sized images after the addition of guest molecules, i.e. ZnPc and ZnPc-L molecules, are shown in Fig. 1 and 2.

Fig. 1a reveals that TSB3,5- $\mathbf{C}_{\mathbf{1 2}}$ molecules form a stable longrange well-ordered 2D honeycomb structure at the air/HOPG interface. Then, it is possible to extract from Fig. $1 \mathrm{~b}$ unit cell parameters associated to the $2 \mathrm{D}$ hexagonal packing ( $a=b=$ $4.2 \mathrm{~nm}$ and $\alpha=60.0^{\circ}$ ). Since they are similar within experimental errors to those reported at the 1-phenyloctane/HOPG interface, ${ }^{17}$ the drop-casting process does not affect the lattice parameters.

To demonstrate the trapping of ZnPc and its derivatives at the air/solid interface, first ZnPc molecules were deposited onto the TSB3,5-C $\mathbf{C}_{12}$ nanoporous monolayer (see the ESI $\dagger$ ). Fig. 2a shows a typical STM image where almost the entire honeycomb structure is filled with ZnPc molecules, appearing as bright spots. These guest molecules are trapped by the host template and they should lie flat on HOPG. It is also possible to observe some features of the underlying nanoporous molecular monolayer. Note that several areas of the HOPG substrate having also a smaller ZnPc coverage rate are observed, probably due the
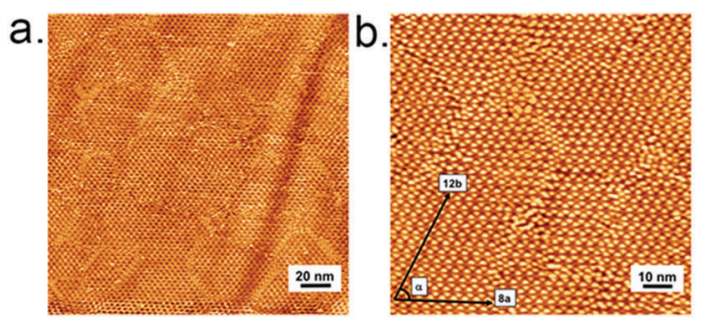

Fig. 1 Well-defined nanoporous monolayer of TSB3,5- $\mathrm{C}_{12}$ molecules deposited on HOPG by drop-casting. (a) $200 \mathrm{~nm} \times 200 \mathrm{~nm}$ long-range STM image and (b) $100 \mathrm{~nm} \times 100 \mathrm{~nm}$ STM image of the supramolecular self-assembly, recorded at the air/HOPG interface in the current mode with $-1.3 \mathrm{~V}$ and $15 \mathrm{pA}$ as the sample bias and tunneling current, respectively. 

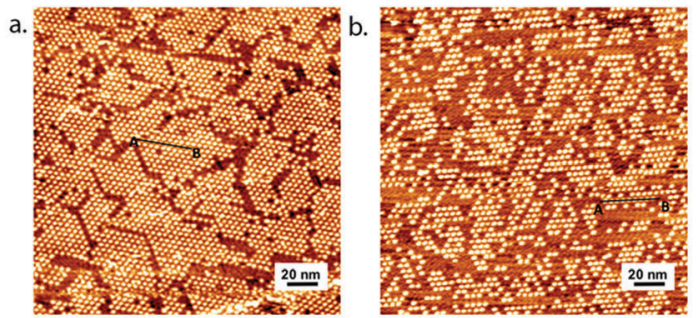

Fig. 2 Nanoporous monolayer of TSB3,5- $\mathrm{C}_{12}$ molecules after the addition, by drop-casting, of guest molecules: (a) ZnPc molecules, $200 \mathrm{~nm} \times$ $200 \mathrm{~nm}$ STM image, (b) ZnPc-L complex molecules, $200 \mathrm{~nm} \times 200 \mathrm{~nm}$ STM image. All images were recorded at the air/HOPG interface in the current mode with $-1.3 \mathrm{~V}$ and $12 \mathrm{pA}$ as the sample bias and tunneling current, respectively.

wettability and the fast drying of chloroform inducing local inhomogeneities.

Now, when the same experiment is performed with ZnPc-L molecules, again the honeycomb structure is filled as shown in Fig. 2b. From the unit cell area of the nanoporous monolayer (Fig. 1b), i.e. $15.3 \mathrm{~nm}^{2}(a=b=4.2 \mathrm{~nm})$, one deduces that the maximum coverage rate for ZnPc or ZnPc-L molecules onto the nanoporous monolayer corresponds to 0.065 molecules per $\mathrm{nm}^{2}$. Thus, the coverage rate is estimated to be $92 \% \pm 2 \%$ and $57 \% \pm$ $2 \%$ for ZnPc (Fig. 2a) and ZnPc-L (Fig. 2b), respectively. Although ZnPc-L molecules are expected to form a protrusion due to the presence of the ligand $\mathbf{L}$, cross-sections corresponding to the black line $\mathrm{AB}$ (Fig. $2 \mathrm{a}$ and $\mathrm{b}$, respectively) show that the apparent heights of ZnPc and ZnPc-L molecules are similar, $0.4 \mathrm{~nm}$ (Fig. S2, ESI†). At this stage, one can only deduce that the nanoporous template is filled, but it is not possible to distinguish between ZnPc and ZnPc-L molecules. Indeed, the observed apparent height is due to the electronic density at the Fermi level, although some authors claim that it should be possible to distinguish between similar molecules through deep, long, and tedious STM investigations whose results at the few nanometer scale are debatable. ${ }^{16}$

In order to complement the information given by STM measurements, another particular challenge for the fabrication of sophisticated out-of-plane functional surfaces is the chemical characterization of the different components used as building blocks. With this aim, resonance Raman spectroscopy was used to chemically probe the functionalization of the HOPG surfaces. The $633 \mathrm{~nm}$ wavelength excitation was chosen, to be in the absorption of the Q band (Fig. S1, ESI $\dagger$ ) of ZnPc and ZnPc-L.

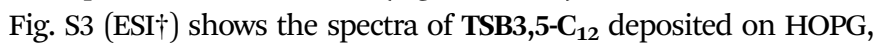
ZnPc and ZnPc-L deposited inside the TSB3,5- $\mathbf{C}_{\mathbf{1 2}}$ network, as well as the spectra of pure $\mathbf{Z n P c}$ and $\mathbf{L}$ molecules. At $633 \mathrm{~nm}$, the Raman spectrum of TSB3,5- $\mathbf{C}_{\mathbf{1 2}}$ deposited on HOPG exhibits only one band at $1582 \mathrm{~cm}^{-1}$, assigned to the G-band of graphite (Fig. S3a, ESI + ). No peak from TSB3,5- $\mathbf{C}_{\mathbf{1 2}}$ is detected since we are outside the absorption range $(\sim 350 \mathrm{~nm})$ of this molecule. When $\mathbf{Z n P c}$ molecules are added into the TSB3,5- $\mathbf{C}_{\mathbf{1 2}}$ templates, additional bands are clearly seen (Fig. S3b, ESI $\dagger$ ). The positions of these bands are in agreement with those of the ZnPc powder (Fig. S3d, ESI $\dagger$ ). Thanks to the resonance effect, we were able to detect the presence of $\mathbf{Z n P c}$ on HOPG, in the pores of the TSB3,5-C $\mathbf{C}_{\mathbf{1 2}}$ network. It is interesting to

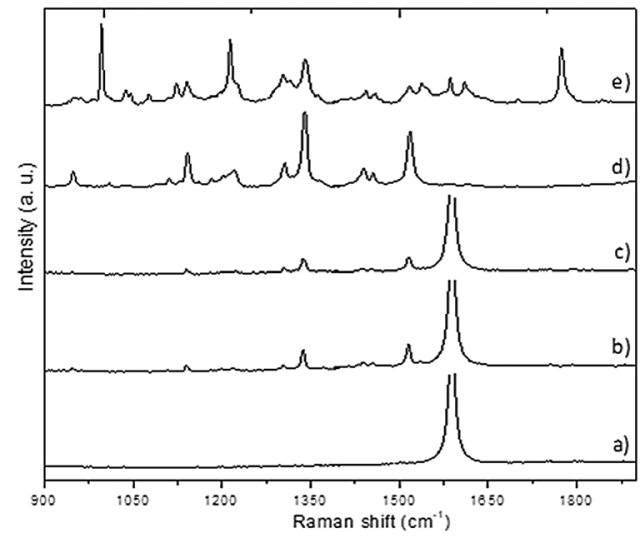

Fig. 3 Raman spectra $(633 \mathrm{~nm})$ of HOPG surfaces with deposits of (a) TSB3,5-C 12 , (b) TSB3,5- $\mathrm{C}_{12}$ and $\mathrm{ZnPc}$, and (c) TSB3,5-C 12 and ZnPc-L. (d and e) The Raman spectra of pure $\mathbf{Z n P c}$ and pure ligand $\mathbf{L}$ powders, respectively.

note that in the literature, even at $633 \mathrm{~nm}$, the Raman signal from Pc on HOPG is usually invisible. ${ }^{27}$ When ZnPc-L molecules are

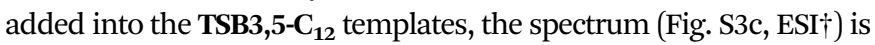
similar to the one obtained after $\mathbf{Z n P c}$ addition. Thus, the Raman signature of the ligand $\mathbf{L}$ is not observed, even the intense bands at 1774, 1213, and $997 \mathrm{~cm}^{-1}$ (Fig. 3). Then, to understand this result, simulated Raman spectra of ZnPc-L at $633 \mathrm{~nm}$ wavelength excitation were computed (see the ESI $\dagger$ for calculation details, geometries, and spectra). The DFT calculations show that, if in the isolated $\mathbf{L}$ ligand, the symmetric stretching of $\mathrm{C}=\mathrm{O}$ bonds $\left(\tilde{\nu} \sim 1780 \mathrm{~cm}^{-1}\right)$ is one of the normal modes of the molecule with the highest Raman activity below $3000 \mathrm{~cm}^{-1}$, it is no longer true in ZnPc-L. Indeed, in the complex, the Raman activity of this normal mode appears about several hundred times lower than that of the normal mode at $\tilde{\nu} \sim$ $1520 \mathrm{~cm}^{-1}$ originating from the Pc moiety. Therefore, it is not surprising that the $\mathrm{C}=\mathrm{O}$ band could not be observed even via resonance Raman spectroscopy experiments for a surface concentration below the monolayer range. The main result of the resonance Raman investigations lies in the observation of the ZnPc signature. This means that its fluorescence is quenched by HOPG and consequently confirms that the Pc macrocycles lie flat on HOPG.

As deduced from the STM characterization and Raman spectroscopy, it is not possible to assure the presence of the ligand $\mathbf{L}$ on top of the $\mathbf{Z n P c}$ hosted inside the pores of the TSB3,5- $\mathbf{C}_{\mathbf{1 2}}$ supramolecular network. Since the deposition of molecular species on a raw surface is known to change its physical properties, we finally decided to track the presence of ligand $\mathbf{L}$ by measuring the HOPG surface energy variations upon modification via our surfaceconfined host-guest approach. This is achieved by measuring the contact angle, $\Theta$, a quantitative measure of the wetting of a solid by a liquid. ${ }^{28}$ Moreover, this macroscopic characterization is compatible with large area substrates. Here, the water contact angles (WCA) of bare HOPG and HOPG after the deposition of TSB3,5$\mathbf{C}_{\mathbf{1 2}}, \mathbf{Z n P c}$, and ZnPc-L were determined (Fig. S3 and Table S1, ESI†). The basal-plane HOPG is known to be slightly hydrophilic, ${ }^{29}$ which is confirmed by the value of $\Theta=84.6^{\circ} \pm 0.8^{\circ}$. Depositing the nonpolar TSB3,5- $\mathbf{C}_{12}$ molecules should increase the hydrophobicity and thus the WCA, as effectively observed $\left(\Theta=91.2^{\circ} \pm 1.4^{\circ}\right)$. In contrast, 
filing the pores of the TSB3,5- $\mathbf{C}_{\mathbf{1 2}}$ network by subsequent addition of ZnPc molecules, soluble in polar solvents, should lead to a decrease of the WCA. This is experimentally confirmed, the contact angle being equal to $\Theta=85.7^{\circ} \pm 1.1^{\circ}$. Thus, angle contact measurement is sensitive enough to estimate the change in the HOPG surface state. Now, by trapping the ZnPc-L complex instead of ZnPc in the 2D porous template, a more significant change is observed as the WCA strongly decreases from $91.2^{\circ} \pm 1.4^{\circ}$ for the TSB3,5- $\mathbf{C}_{\mathbf{1 2}}$ network to $78.1^{\circ} \pm 1.3^{\circ}$. These results demonstrate the filling of the pores by a species dissimilar to ZnPc that modifies drastically the hydrophilicity of the entire substrate. We can infer that this molecule is the 3D ZnPc-L complex, its elbow structure evidenced by modeling favoring the hydrophilicity. Indeed, the two $\mathrm{C}=\mathrm{O}$ bonds are available for H-bonding with water molecules on the one hand, and, in addition, within this geometry, ZnPc-L was computed to have a permanent dipolar moment of 7.2 $\mathrm{D}$ on the other hand. Finally, the above hydrophilicity tuning demonstrates the HOPG functionalization.

To summarize, we have developed a new method to trap a functional 3D ZnPc-L complex into a large 2D nanoporous template. This facile process combines the surface-confined host-guest chemistry and drop-casting method. We describe an unusual approach relying on the large-scale observation of the functionalized surface allowing the use of several experimental techniques such as STM at the air/solid interface, resonance Raman spectroscopy, and contact angle measurements. By overlapping the different results, it is possible to reliably prove the controlled immobilization of the building block molecules at a long range. Due to the versatility of the ligand complex chemistry, our manipulable platform could be used for a new generation of clickable surfaces for potential applications in e.g. nanophotonics or biosensing.

This work was supported by the LabEx MiChem, part of French state funds managed by the ANR within the "Investissements d'Avenir" program under reference ANR-11-IDEX-0004-02 and the Global Research Laboratory (GRL) through the National Research Foundation of Korea (NRF) funded by the Ministry of Science, ICT \& Future Planning (no. 2016K1A1A2912753). We thank Y. Berger (MONARIS) for his helpful technical support.

\section{Conflicts of interest}

There are no conflicts to declare.

\section{Notes and references}

1 J. Barth, G. Costantini and K. Kern, Nature, 2005, 437, 671; C.-A. Palma, M. Cecchini and P. Samori, Chem. Soc. Rev., 2012, 41, 3713; S. Mali, J. Adisoejoso, E. Ghijsens, I. De Cat and S. De Feyter, Acc. Chem. Res., 2012, 45, 1309.

2 T. Yokoyama, S. Yokoyama, T. Kamikado, Y. Okuno and S. Mashiko, Nature, 2001, 413, 619; S. De Feyter and F. C. De Schryver, J. Phys. Chem. B, 2005, 109, 4290.

3 L. Sosa-Vargas, E. Kim and A. J. Attias, Mater. Horiz., 2017, 4, 570; J. A. A. W. Elemans, S. B. Lei and S. De Feyter, Angew. Chem., Int. Ed., 2009, 8, 7298.
4 D. Bonifazi, S. Mohnani and A. Llanes-Pallas, Chem. - Eur. J., 2009, 15, 7004; T. Kudernac, S. Lei, J. A. A. W. Elemans and S. De Feyter, Chem. Soc. Rev., 2009, 38, 402; J. Teyssandier, S. De Feyter and K. S. Mali, Chem. Commun., 2016, 52, 11465.

5 S. Stepanow, M. Lingenfelder, A. Dmitriev, H. Spellmann, E. Delvigne, N. Lin, X. Deng, C. Cai, J. V. Barth and K. Kern, Nat. Mater., 2004, 3, 229; H. L. Zhang, W. Chen, H. Huang, L. Chen and A. T. S. Wee, J. Am. Chem. Soc., 2008, 130, 2720; M. Blunt, X. Lin, M. d. C. Gimenez-Lopez, M. Schroder, N. R. Champness and P. H. Beton, Chem. Commun., 2008, 2304.

6 J. Adisoejoso, K. Tahara, S. Okuhata, S. Lei, Y. Tobe and S. De Feyter, Angew. Chem., Int. Ed., 2009, 48, 7353.

7 G. Schull, L. Douillard, C. Fiorini-Debuisschert, F. Charra, F. Mathevet, D. Kreher and A. J. Attias, Nano Lett., 2006, 6, 1360.

8 K. Tahara, K. Nakatani, K. Iritani, S. De Feyter and Y. Tobe, ACS Nano, 2016, 10, 2113.

9 K. Tahara, K. Inukai, J. Adisoejoso, H. Yamaga, T. Balandina, M. O. Blunt, S. De Feyter and Y. Tobe, Angew. Chem., Int. Ed., 2013, 52, 8373.

10 X. Zhang, Y. Shen, S. Wang, Y. Guo, K. Deng, C. Wang and Q. Zeng, Sci. Rep., 2012, 2, 742.

11 M. O. Blunt, J. C. Russell, M. d. C. Gimenez-Lopez, N. Taleb, X. Lin, M. Schröder, N. R. Champness and P. H. Beton, Nat. Chem., 2011, 3,74 .

12 B. Baisch, D. Raffa, U. Jung, O. M. Magnussen, C. Nicolas, J. Lacour, J. Kubitschke and R. Herges, J. Am. Chem. Soc., 2009, 131, 442; M. Hammerich, T. Rusch, N. R. Krekiehn, A. Bloedorn, O. M. Magnussen and R. Herges, ChemPhysChem, 2016, 17, 1870.

13 P. Du, M. Jaouen, A. Bocheux, C. Bourgogne, Z. Han, V. Bouchiat, D. Kreher, F. Mathevet, C. Fiorini-Debuisschert, F. Charra and A.-J. Attias, Angew. Chem., Int. Ed., 2014, 53, 10060; S. Le Liepvre, P. Du, D. Kreher, F. Mathevet, A.-J. Attias, C. Fiorini-Debuisschert, L. Douillard and F. Charra, ACS Photonics, 2016, 3, 2291.

14 J. M. Gottfried, Surf. Sci. Rep., 2015, 70, 259.

15 T. Ye, T. Takami, R. Wang, J. Jiang and P. S. Weiss, J. Am. Chem. Soc., 2006, 128, 10984; W. Auwärter, D. Écija, F. Klappenberger and J. V. Barth, Nat. Chem., 2015, 7, 105.

16 O. P. H. Vaughan, F. J. Williams, N. Bampos and R. M. Lambert, Angew. Chem., Int. Ed., 2006, 45, 3779; J. Visser, N. Katsonis, J. Vicario and B. L. Feringa, Langmuir, 2009, 25, 5980.

17 D. Bleger, D. Kreher, F. Mathevet, A. J. Attias, G. Schull, A. Huard, L. Douillard, C. Fiorini-Debuischert and F. Charra, Angew. Chem., Int. Ed., 2007, 46, 7404; G. Schull, L. Douillard, C. Fiorini-Debuisschert, F. Charra, F. Mathevet, D. Kreher and A. J. Attias, Adv. Mater., 2006, 18, 2954; C. Arrigoni, G. Schull, D. Bléger, L. Douillard, C. FioriniDebuisschert, F. Mathevet, D. Kreher, A.-J. Attias and F. Charra, J. Phys. Chem. Lett., 2010, 1, 190.

18 G. Schull, PhD thesis, École normale supérieure de Cachan, 2006.

19 T. Inose, D. Tanaka, O. Ivasenko, K. Tahara, S. De Feyter, Y. Tobe, H. Tanaka and T. Ogawa, Chem. Lett., 2016, 45, 286.

20 D. P. Nair, M. Podgórski, S. Chatani, T. Gong, W. Xi, C. R. Fenoli and C. N. Bowman, Chem. Mater., 2014, 26, 724; A. D. Baldwin and K. L. Kiick, Bioconjugate Chem., 2011, 22, 1946.

21 S. K. Sinha and S. K. Shrivastava, Bioorg. Med. Chem. Lett., 2013, 23, 2984.

22 H. Wurm, W. Regel and M. L. Hallensleben, Makromol. Chem., 1979, 180, 1581; F. Severini, M. Pegoraro, G. Ricca, G. Audisio, D. Dainese and R. Gallo, Polym. Int., 1990, 23, 23.

23 J. Kötteritzsch, S. Stumpf, S. Hoeppener, J. Vitz, M. D. Hager and U. S. Schubert, Macromol. Chem. Phys., 2013, 214, 1636.

24 R. Díaz-Torres and S. Alvarez, Dalton Trans., 2011, 40, 10742.

25 P. Kasprzycki, L. Sobotta, S. Lijewski, M. Wierzchowski, T. Goslinski, J. Mielcarek, C. Radzewicz and P. Fita, Phys. Chem. Chem. Phys., $2017,19,21390$.

26 M. E. El-Khouly and S. Fukuzumi, Photochem. Photobiol. Sci., 2016, 15, 1340; Y. Rio, W. Seitz, A. Gouloumis, P. Vázquez, J. L. Sessler, D. M. Guldi and T. Torres, Chem. - Eur. J., 2010, 16, 1929.

27 X. Ling, L. Xie, Y. Fang, H. Xu, H. Zhang, J. Kong, M. S. Dresselhaus, J. Zhang and Z. Liu, Nano Lett., 2010, 10, 553.

28 D. Y. Kwok, T. Gietzelt, K. Grundke, H.-J. Jacobasch and A. W. Neumann, Langmuir, 1997, 13, 2880.

29 Y. Wei and C. Q. Jia, Carbon, 2015, 87, 10. 